\title{
Constitutive Parameters Determination of Bi-anisotropic Metamaterials using a Waveguide Method
}

\author{
U. C. Hasar ${ }^{1}$, M. Bute ${ }^{1}$, A. Muratoglu ${ }^{1}$, J. J. Barroso ${ }^{2}$, T. Karacali ${ }^{3}$, and M. Ertugrul ${ }^{3}$ \\ ${ }^{1}$ University of Gaziantep, Dept. Electrical and Electronics Eng., Gaziantep 27310, Turkey \\ ${ }^{2}$ National Institute for Space Research, Associated Plasma Lab., 12227-010 São José dos Campos, SP, Brazil \\ ${ }^{3}$ Ataturk University, Dept. Electrical and Electronics Eng., Erzurum 25240, Turkey
}

\begin{abstract}
A retrieval method is proposed for determination of electromagnetic properties of bi-anisotropic metamaterial (MM) slabs inserted in a waveguide. For this goal, forward and backward reflection and transmission scattering (S-) parameters of a configuration where the slab is positioned into a waveguide are first derived and then a procedure for extraction of electromagnetic properties of this slab is presented. We performed full-wave electromagnetic simulations on the bi-anisotropic MM slab with split-ringresonators to validate the proposed method.
\end{abstract}

Index Terms - Metamaterial, electromagnetic parameter, retrieval, bi-anisotropy.

\section{INTRODUCTION}

In 1968, Veselago [1] opened the era of new artificial materials and developed the concept of a material with a negative refractive index, which simultaneously exhibits negative relative complex permittivity $\left(\varepsilon_{r}\right)$ and negative relative complex permeability $\left(\mu_{r}\right)$ with negative real parts over a desired frequency range [2]. Pendry et al. proposed two composite components for the realization of such materials as: a) arrays of thin metal wires resulting in a negative $\varepsilon_{r}$ [3] and $\mathrm{b}$ ) arrays of split-ring resonators (SRR) giving in negative $\mu_{r}$ [4]. These materials are referred to as metamaterials (MMs) and follow the left-hand rule. MMs have very interesting electromagnetic characteristics leading to direct applications in electromagnetic cloaks and concentrators, new types of antennas, sub-wavelength waveguides, and filters. Thus, there is a huge demand for electromagnetic characterization of these materials for the above applications.

Depending on incident wave configuration, an isotropic MM slab can gain a bi-anisotropic property. A bianisotropic MM slab has non-identical forward and backward wave impedances, forward and backward reflection scattering (S-) parameters, and forward and backward powers [2],[5]. Many retrieval techniques based on scattering (S-) parameter measurements have been proposed for electromagnetic characterization of MM slabs in the literature [2],[6]-[12]. Among these techniques, the waveguide method [10] seems to be superior in that it does not require a large periodic arrangement in transverse dimension and generates relatively higher sensitivity due to confinement of electromagnetic energy within guide walls. However, this method was only

applied for electromagnetic characterization of isotropic MM slabs. In this study, we propose another waveguide

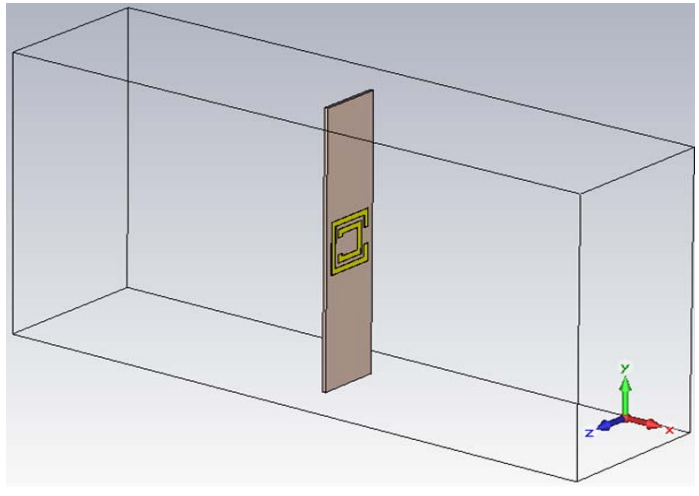

Fig. 1. Arrangement of one split-ring-resonator within a waveguide. Input and output ports are perpendicular to the $\mathrm{z}$ direction, and PEC (perfect electric conductor) boundary conditions are applied to $\mathrm{xz}$ and $\mathrm{yz}$ planes.

method for determination of electromagnetic parameters of bi-anisotropic MM slabs.

\section{PROPOSED METHOD}

As depicted in Fig. 1, in the waveguide is positioned a MM slab with the gap-bearing sides parallel to the $y$ direction. It is assumed that fundamental mode $\left(\mathrm{TE}_{10}\right)$ propagates along the $z$ direction from air onto the SRR MM slab. Since the incident electric field is parallel to the $y$ axis, $\mathrm{E}_{\mathrm{y}}$ produces an unbalanced current distribution in the rings, inducing a magnetic dipole moment in the $\mathrm{x}$ direction. Due to the asymmetry of the inner and outer rings (Fig. 2), the accompanying magnetic field $\mathrm{H}_{\mathrm{x}}$ induces an electric dipole in the $y$ direction [5]. Therefore, electric and magnetic fields will be coupled, producing bi-anisotropic effects described by the magneto-electric coupling coefficient $\xi_{0}$.

Applying necessary boundary conditions (vanishing of tangential electric fields over guide walls), expressions of electric and magnetic fields within air (superscript ' 0 ') and slab (superscript 's') regions are obtained for the time reference in the form $\exp (-i \omega t)$ as

$$
\begin{gathered}
E_{y}^{0}(x, z)=\sin \left(\beta_{x 0} x\right)\left[K_{1} e^{+i \beta_{z 0} z}+K_{2} e^{-i \beta_{z 0} z}\right], \\
H_{x}^{0}(x, z)=\frac{\sin \left(\beta_{x 0} x\right)}{i \omega \mu_{0}}\left[-i \beta_{z 0} K_{1} e^{+i \beta_{z 0} z}+i \beta_{z 0} K_{2} e^{-i \beta_{z 0} z}\right], \\
H_{z}^{0}(x, z)=\frac{\beta_{x 0}}{i \omega \mu_{0}} \cos \left(\beta_{x 0} x\right)\left[K_{1} e^{+i \beta_{z 0} z}+K_{2} e^{-i \beta_{z 0} z}\right], \\
E_{y}^{s}(x, z)=\sin \left(\beta_{x 0} x\right)\left[K_{3} e^{+i \beta_{z s} z}+K_{4} e^{-i \beta_{z s} z}\right],
\end{gathered}
$$




$$
\begin{gathered}
H_{x}^{s}(x, z)=\frac{\sin \left(\beta_{x 0} x\right)}{\mu_{0} \mu_{1}}\left\{\begin{array}{l}
-K_{3}\left(\frac{\beta_{z s}}{\omega}+i \frac{\xi_{0}}{c}\right) e^{+i \beta_{z s} z} \\
+K_{4}\left(\frac{\beta_{z s}}{\omega}-i \frac{\xi_{0}}{c}\right) e^{-i \beta_{z s} z}
\end{array}\right\}, \\
H_{z}^{s}(x, z)=\frac{\beta_{0 x}}{i \omega \mu_{0} \mu_{2}} \cos \left(\beta_{0 x} x\right)\left[K_{3} e^{+i \beta_{s z} z}+K_{4} e^{-i \beta_{s z} z}\right],
\end{gathered}
$$

where $K_{1}, K_{2}, K_{3}$, and $K_{4}$ are constants and

$$
\begin{aligned}
& \beta_{x 0}=\pi / a, \quad \beta_{z 0}=\sqrt{\beta_{0}^{2}-\beta_{x 0}^{2}}, \\
& \beta_{z S}=\sqrt{k_{0}^{2}\left(\varepsilon \mu_{1}-\xi_{0}^{2}\right)-\frac{\mu_{1}}{\mu_{2}} \beta_{x 0}^{2}} .
\end{aligned}
$$

Here, $\varepsilon$ is the relative complex permittivity in $y$ direction, $\mu_{1}$ and $\mu_{2}$ are respectively the relative complex permeability in $x$ and $z$ directions; $\mu_{0}$ is the magnetic permeability of free-space; $k_{0}$ is the free-space wavenumber; and $a$ is the broader dimension of the guide. It is noticed that when magneto-electric coupling coefficient $\xi_{0}$ goes to zero (isotropic MM slab case), the expression of $\beta_{z S}$ reduces to a form similar to (3) in [10], substantiating the analysis of forward problem.

Using the electric and magnetic field expressions in (1)(6) and enforcing appropriate boundary conditions over the interface of air-MM slab (continuity of tangential components of electric and magnetic fields), forward and backward reflection and transmission S-parameters are derived as

$$
\begin{gathered}
S_{11}=\frac{\Gamma_{1}\left(1-T^{2}\right)}{1-\Gamma_{1} \Gamma_{2} T^{2}}, \quad S_{22}=\frac{\Gamma_{2}\left(1-T^{2}\right)}{1-\Gamma_{1} \Gamma_{2} T^{2}}, \\
S_{21}=S_{12}=\frac{\left(1-\Gamma_{1} \Gamma_{2}\right) T}{\left(1-\Gamma_{1} \Gamma_{2} T^{2}\right)},
\end{gathered}
$$

where $d$ is the thickness of the MM slab

$$
\begin{gathered}
\Gamma_{1}=\frac{z_{w s}^{+}-1}{z_{w s}^{+}+1}, \quad \Gamma_{2}=\frac{z_{w s}^{-}-1}{z_{w s}^{-}+1}, \quad T=\exp \left(+i \beta_{s z} d\right), \\
z_{w s}^{+}=\frac{\mu_{1} \beta_{0 z}}{\beta_{s z}+i k_{0} \xi_{0}}, \quad z_{w s}^{-}=\frac{\mu_{1} \beta_{0 z}}{\beta_{s z}-i k_{0} \xi_{0}} .
\end{gathered}
$$

Here, $z_{w s}^{+}$and $z_{w s}^{-}$are the wave impedances of the slab in forward $(+z)$ and backward $(-z)$ directions.

To determine electromagnetic properties of the bianisotropic MM slab, we apply the following approach. First, we find $z_{w s}^{+}$and $z_{w s}^{-}$as [12]

$$
\begin{array}{lc}
z_{w s}^{+}=\frac{-\Lambda_{2} \mp \sqrt{\Lambda_{2}^{2}-4 \Lambda_{1} \Lambda_{3}}}{2 \Lambda_{1}}, & z_{w s}^{-}=\frac{z_{w s}^{+}+\Lambda_{4}}{1+z_{w s}^{+} \Lambda_{4}}, \\
\Lambda_{1}=S_{21}^{2}-\left(1-S_{11}\right)\left(1-S_{22}\right), & \Lambda_{2}=2\left(S_{11}-S_{22}\right), \\
\Lambda_{3}=\left(1+S_{11}\right)\left(1+S_{22}\right)-S_{21}^{2}, & \Lambda_{4}=\frac{S_{11}-S_{22}}{S_{11}+S_{22}} .
\end{array}
$$

Then, we determine $T$ using (11) as

$$
T=\frac{\Gamma_{1}\left(1-\Gamma_{1} \Gamma_{2}\right) S_{21}}{\left(1-\Gamma_{1} \Gamma_{2}\right)\left(\Gamma_{1}-S_{11} \Gamma_{1} \Gamma_{2}\right)} .
$$

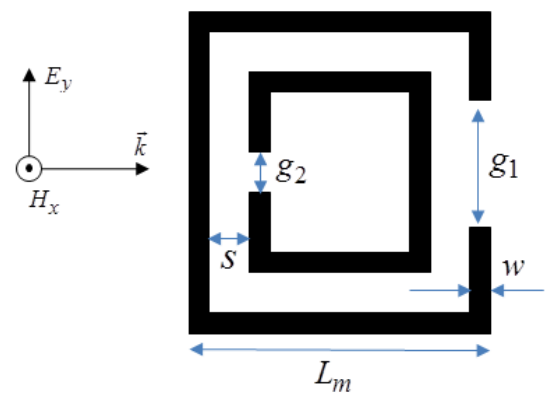

Fig. 2. Geometrical details of the analyzed split-ring-resonator.

Next, we obtain $\xi_{0}$ and $\mu_{1}$ as

$$
\xi_{0}=\frac{1}{k_{0} d} \ln (T)\left(\frac{z_{w s}^{+}-z_{w s}^{-}}{z_{w s}^{+}+z_{w s}^{-}}\right), \quad \mu_{1}=z_{w s}^{+}\left(\frac{\ln (T)-k_{0} \xi_{0} d}{i \beta_{0 z} d}\right) .
$$

If we assume that magnetic field in $z$ direction does not produce any magnetic resonance, we can then set $\mu_{2}=1$, yielding the determination of the last unknown $\varepsilon_{3}$ as

$$
\varepsilon_{3}=\frac{1}{\mu_{1}}\left[\frac{\xi_{0}^{2} k_{0}^{2}-\frac{[\ln (T)]^{2}}{d^{2}}+\mu_{1}\left(\frac{\pi}{a}\right)^{2}}{k_{0}^{2}}\right] .
$$

As determined above, the permittivity describes the excitation of electric dipoles by the electric field; the permeability describes the excitation of magnetic dipoles by the magnetic field; the magnetoelectric coupling parameter describes the excitation of magnetic dipoles by the electric field and the excitation of electric dipoles by the magnetic field.

\section{VALIDATION}

For validation of our proposed method, we consider a slab of square split-ring-resonator (SRR) shown in Fig. 2. Its geometrical and electromagnetic parameters are as follows.

The outer SRR has a side of $L_{m}=1.9 \mathrm{~mm}$ and both rings have an angular line width of $w=0.15 \mathrm{~mm}$. Separation distance between SRRs is $s=0.25 \mathrm{~mm}$ and air gaps for the rings are $g_{1}=1.0 \mathrm{~mm}$ and $g_{2}=0.5 \mathrm{~mm}$. Substrate has a dielectric constant 4.3 and thickness $t_{s}=3.0 \mathrm{~mm}$. Copper material $35 \mu \mathrm{m}$ thick is used for tracks of SRRs.

We use the CST Microwave Studio for simulation of Sparameters of the configuration in Fig. 1 with cell parameters in Fig. 2 and slab thickness $\mathrm{d}=2.0 \mathrm{~mm}$. Perfect electric conductors are assumed to be present over $y z$ and $x z$ planes to be coincident with the guide walls. In our analysis, an X-band $(22.86 \mathrm{~mm}$ and $10.16 \mathrm{~mm})$ waveguide is considered. It is assumed that waveguide ports are perpendicular to the $z$ axis (direction of wave propagation) for obtaining input/output. Details of our simulations can be found in [5] and [12].

Fig. 3 shows the frequency response of forward and backward S-parameters of the SRR MM slab in Fig. 1. It is seen from Fig. 3(a) and Fig. 3(b) that forward and backward reflection S-parameters of this slab are different from one another especially over the frequency range $10.2-10.8$ 

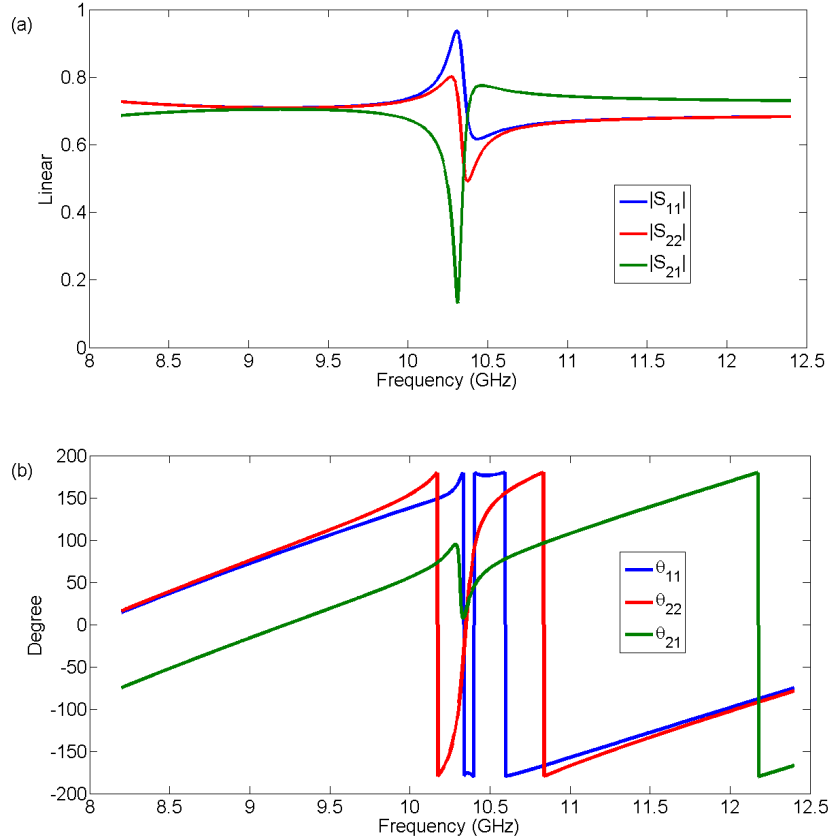

Fig. 3. Frequency response of (a) magnitude and (b) phase of forward and backward reflection and transmission S-parameters of the SRR MM slab in Fig. 1 with geometrical details in Fig. 2.

$\mathrm{GHz}$ where $\mathrm{MM}$ slab is in resonance due to metallic inclusions. It is expected that bi-anisotropic coupling coefficient over this frequency range becomes far different from zero. Considering the simulated S-parameters in Fig. 3 and assuming that $\mu_{2}=1$, we then applied our proposed method. Figs. 4(a)-4(c) demonstrate the real and imaginary parts of the extracted constitutive parameters of the MM slab. It is seen from Fig. 4(a) that magneto-electric coupling coefficient $\xi_{0}$ reaches a non-zero value around the frequency range 10.2-10.8 GHz. We note from Fig. 4(b) and 4(c) that imaginary parts of relative complex permittivity and relative complex permeability of the analyzed MM slab are greater than zero over whole frequency band. Moreover, it is seen from Fig. 4(b) and 4(c) that their values considerably increase around the resonance region (10.2 $10.8 \mathrm{GHz}$ ), exemplifying a common behavior of resonators. Finally, we notice that, aside from the resonance region $(10.2-10.8 \mathrm{GHz})$, real parts of relative complex permittivity and complex permeability are asymptotically approaching 1 and 4.3 which are consistent with the electromagnetic properties of the substrate housing metallic tracks.

\section{CONCLUSIONS}

We proposed a waveguide method for extraction of electromagnetic properties of bi-anisotropic MM slabs. We first derived the expressions of forward and backward reflection and transmission S-parameters of a typical bianisotropic MM slab by applying necessary boundary conditions and then proposed a procedure for retrieval of electromagnetic properties of this slab. We validated our derivations by considering the case that magneto-coupling coefficient goes to zero and by performing some simulations
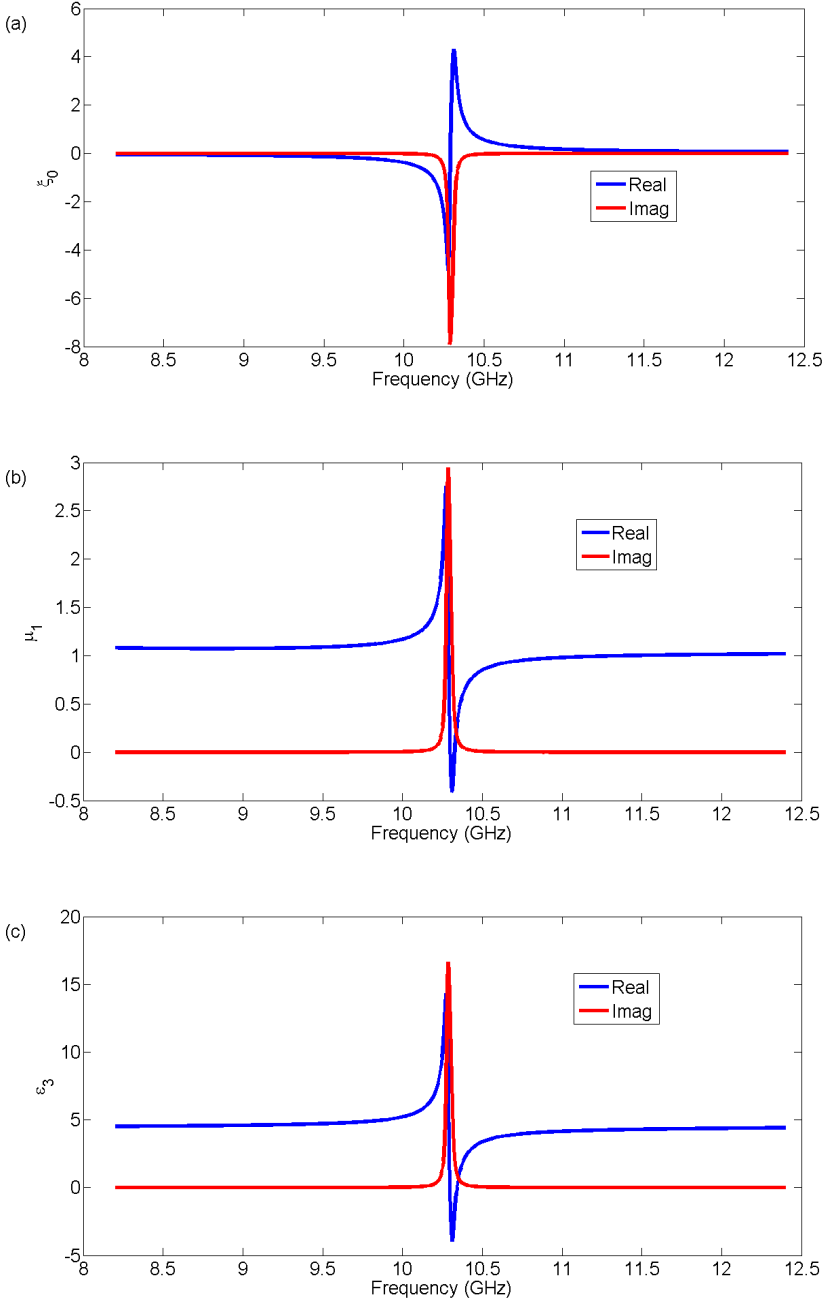

Fig. 4. Real and imaginary parts of retrieved (a) magneto-electric coupling coefficient, (b) relative complex permittivity in y direction, and (c) relative complex permeability in $\mathrm{x}$ direction of the MM slab in Fig. 1 with geometrical details in Fig. 2.

of a bi-anisotropic MM slab composed of split-ringresonators.

\section{ACKNOWLEDGEMENT}

U. C. Hasar, M. Bute, A. Muratoglu, T. Karacali, and M. Ertugrul express their thanks to the Scientific and Technological Research Council of Turkey under the project Grant Number 112R032 for supporting this study. U. C. Hasar also sends his special thanks to the Science Academy of Turkey for the Young Scientist award in 2014.

\section{REFERENCES}

[1] V. G. Veselago, "The electrodynamics of substances with simultaneously negative values of $\varepsilon$ and $\mu$,"Sov. Phys. USPEKHI, vol. 10, pp. 509-514, 1968.

[2] Z. Li, K. Aydin, and E. Ozbay, "Determination of the effective constitutive parameters of bianisotropic metamaterials from reflection and transmission coefficients," Phys. Rev. E, vol. 79, 026610, 2009.

[3] J. B. Pendry, A. J. Holden, W. J. Stewart, and I. Youngs, "Extremely low frequency plasmons in metallic mesostructures," Phys. Rev. Lett., vol. 76, pp. 4773-4776, 1996. 
[4] J. B. Pendry, A. J. Hold, D. J. Robbins, and W. J. Stewart, "Magnetism from conductors and enhanced nonlinear phenomena," IEEE Trans. Microw. Theory Tech., vol. 47, pp. 2075-2084, 1999.

[5] U. C. Hasar, M. Bute, J. J. Barroso, C. Sabah, Y. Kaya, M. Ertugrul, "Power analysis of multilayer structures composed of conventional materials and bianisotropic metamaterial slabs", J. Opt. Soc. Am. B, vol. 31, 939-947, 2014.

[6] D. R. Smith, S. Schultz, P. Markos, and C. M. Soukoulis, "Determination of effective permittivity and permeability of metamaterials from reflection and transmission coefficients," Phys. Rev. B, vol. 65, 195104, 2002.

[7] M. S. Bombay, and O. M. Ramahi, "Open-ended coaxial line probes with negative permittivity materials," IEEE Trans. Antennas Propagat., vol. 59, pp. 1765-1769, 2011.

[8] X. Chen, T. M. Grzegorczyk, B. -I. Wu, J. Pacheco, J. A. Kong, "Robust method to retrieve the constitutive effective parameters of metamaterials," Phys. Rev. E, vol. 70, 016608, 2004.

[9] X. Chen, B. -I. Wu, J. A. Kong, T. M. Grzegorczyk, "Retrieval of the effective constitutive parameters of bianisotropic metamaterials," Phys. Rev. E, vol. 71, 046610, 2005.

[10] H. Chen, J. Zhang, Y. Bai, Y. Luo, L. Ran, Q. Jiang, J. A. Kong, "Experimental retrieval of the effective parameters of metamaterials based on a waveguide method," Opt. Express, vol. 14, 12944-12949, 2006.

[11] D. R. Smith, D. C. Vier, T. Koschny, C. M. Soukoulis, "Electromagnetic parameter retrieval from inhomogenous metamaterials," Phys. Rev. E, vol. 71, 036617, 2005.

[12] U. C. Hasar, J. J. Barroso, C. Sabah, Y. Kaya, M. Ertugrul, "Stepwise technique for accurate and unique retrieval of electromagnetic properties of bianisotropic metamaterials", $J$. Opt. Soc. Am. B, vol. 30, 1058-1068, 2013. 